\title{
CONSCIÊNCIA DO PRÓPRIO DESVIO DE FALA E PROCESSAMENTO AUDITIVO NO DESVIO FONOLÓGICO
}

\author{
Awareness of their own speech impairment \\ and auditory processing in phonological disorder
}

\author{
Roberta Freitas Dias( ${ }^{(1)}$, Victor Gandra Quintas(2), Roberta Michelon Melo(3), \\ Helena Bolli Mota(4), Carolina Lisbôa Mezzomo ${ }^{(5)}$
}

\section{RESUMO}

Tema: a habilidade de reconhecimento do próprio desvio de fala e sua possível relação com o processamento auditivo em crianças com diagnóstico de desvio fonológico. Procedimentos: participaram do estudo oito sujeitos com diagnóstico de desvio fonológico e idades 5:0;26 e 7:7;2. O sistema fonético/fonológico foi avaliado por meio do instrumento - Avaliação Fonológica da Criança. Aplicou-se ainda o teste de consciência do próprio desvio de fala, o qual tem por finalidade fazer com que a criança ouça e julgue os desvios existentes em sua própria fala. Para avaliação do processamento auditivo, utilizou-se a Avaliação Simplificada do Processamento Auditivo (Triagem), e os testes especiais de Fala no Ruído e o Teste de Dissílabos Alternados. Resultados: todos os sujeitos obtiveram resultados abaixo do esperado, com valores abaixo de $50 \%$ do valor máximo possível nos testes de processamento auditivo. Quanto à consciência do próprio desvio de fala, todos os sujeitos apresentaram esta habilidade, mas em valores bastante variáveis ainda que o desempenho dos testes do processamento auditivo não tenham sido satisfatórios. Este achado sugere que as crianças neste período (5-7 anos), mesmo apresentando dificuldade para entender ou interpretar o que ouvem, são capazes de perceber os erros que apresentam na fala. Conclusão: a consciência do próprio desvio de fala está presente nos sujeitos com desvio fonológico, independentemente do desempenho apresentado em tarefas do processamento auditivo.

DESCRITORES: Distúrbios da Fala; Testes Auditivos; Percepção Auditiva; Discriminação da Fala; Criança

(1) Fonoaudióloga; Professora Substituta do Curso de Fonoaudiologia da Universidade Federal de Santa Maria (UFSM), Mestre em Distúrbios da Comunicação Humana pela UFSM, Santa Maria, RS, Brasil.

(2) Fonoaudiólogo clínico em Contagem, MG, Brasil; Mestre em Distúrbios da Comunicação Humana pela UFSM, Santa Maria, RS, Brasil.

(3) Fonoaudióloga; Bolsista CAPES (Coordenação de Aperfeiçoamento de Pessoal de Nível Superior); Mestranda em Distúrbios da Comunicação Humana pela UFSM, Santa Maria, RS, Brasil.

(4) Fonoaudióloga; Professora Associada do Curso de Fonoaudiologia da UFSM, Santa Maria, RS, Brasil; Doutora em Linguística Aplicada pela Pontifícia Universidade Católica do Rio Grande do Sul - PUCRS -, Porto Alegre, RS, Brasil.

(5) Fonoaudióloga; Professora Adjunto do Curso de Fonoaudiologia da Universidade Federal de Santa Maria - UFSM, Santa Maria, RS, Brasil; Doutora em Linguística Aplicada pela Pontifícia Universidade Católica do Rio Grande do Sul - PUCRS -, Porto Alegre, RS, Brasil.

Conflito de interesses: inexistente

\section{INTRODUÇÃO}

Os desvios fonológicos são alterações de fala sem etiologia orgânica aparente, em que há desordem linguística na representação mental dos sons da fala ${ }^{1,2}$. Assim como no desenvolvimento fonológico típico, nos desvios fonológicos é possível encontrar indícios de um conhecimento fonológico da língua-alvo, maior, mais maduro e mais adequado, do que esses sujeitos são capazes de evidenciar nas suas produções ${ }^{3-5}$.

Existem crianças com desvio fonológico que podem ter consciência da sua fonologia desviante, 0 que demonstra que elas podem ter acesso a representações fonológicas normais. Essa consciência leva as crianças a fazerem tentativas de transmitir o sentido correto das palavras, o que não quer dizer 
que elas tenham considerado a estrutura linguística ou realizado manipulações conscientes das formas para transmitir o seu significado ${ }^{6}$.

Considerando que crianças com desvio fonológico podem reconhecer suas trocas de fala, entende-se que elas podem ter consciência do próprio desvio ${ }^{7}$.

Assim, pode-se assumir que a percepção de certos sons por crianças com desvio fonológico pode ser melhor do que a sua produção ${ }^{8,9}$ o que indica ser necessária uma maturação do sistema auditivo, já que esta é a principal via de entrada para a aquisição fonológica e sua percepção, sobretudo o processamento auditivo, que pode estar diretamente relacionado às dificuldades na linguagem oral, como as observadas no desvio fonológico ${ }^{10-13}$.

O processamento auditivo pode ser definido como mecanismos e processos do sistema auditivo responsáveis pelos fenômenos comportamentais, tais como, localização sonora, discriminação auditiva, reconhecimento auditivo, resolução e ordenação temporal, desempenho auditivo com sinais acústicos competitivos e desempenho auditivo com sinais acústicos degradados ${ }^{14}$.

A hipótese incentivadora da presente pesquisa consiste no fato de que, a partir da língua-alvo, algumas crianças com desvio fonológico podem apresentar melhor percepção (discriminação, reconhecimento do som, etc.) dos segmentos linguísticos do que a capacidade de produção dos mesmos. Além disso, tanto pela consciência do próprio desvio de fala como pelo processamento auditivo envolverem alguns mecanismos e processos auditivos em comum, supõe-se que estes possam apresentar algumas relações semelhantes no processo de percepção dos sons.

Assim, os resultados obtidos com este trabalho pretendem colaborar para um melhor entendimento da relação entre o desvio fonológico e outras habilidades linguísticas, contribuindo para a realização de prognósticos e condutas terapêuticas de fala mais completas e efetivas.

A partir do exposto, este estudo tem como objetivos, investigar o desempenho de crianças com diagnóstico de desvio fonológico no teste de consciência do próprio desvio de fala e na avaliação do processamento auditivo. Bem como, identificar as prováveis relações da consciência do próprio desvio de fala com o processamento auditivo destas crianças.

\section{APRESENTAÇÃO DO CASO}

Realizou-se um estudo do tipo transversalquantitativo, em que participaram oito sujeitos, seis meninos e duas meninas, com idades entre 5:0;26 e 7:7;2 e com diagnóstico de desvio fonológico.

Para que fizessem parte da amostra, os sujeitos deveriam apresentar os seguintes critérios de inclusão: ter autorização dos pais/responsáveis para a participação na pesquisa por meio da assinatura do Termo de Consentimento Livre Esclarecido; o assentimento da criança em participar do estudo; ter idades entre 5:0 a 7:11;29; apresentar diagnóstico de desvio fonológico; não ter recebido ou estar recebendo algum tipo de terapia fonoaudiológica; não apresentar alterações nas avaliações da triagem fonoaudiológica; não apresentar presença de comprometimentos evidentes nos aspectos neurológico, cognitivo ou psicológico e não apresentar alterações na triagem auditiva.

Para a realização do diagnóstico de desvio fonológico e seleção da amostra, os sujeitos passaram por uma triagem fonoaudiológica em que foram realizadas: avaliação do sistema estomatognático, avaliação da linguagem e da fala, além de triagem auditiva.

O sistema fonético/fonológico foi avaliado por meio do instrumento Avaliação Fonológica da Criança (AFC) ${ }^{1}$ que possibilita a obtenção de uma amostra de fala, contendo todos os fones contrastivos do português brasileiro em todas as posições que podem ocorrer em relação à estrutura da sílaba.

Depois de realizada a triagem fonoaudiológica, procedeu-se com a coleta dos dados. Inicialmente realizou-se o teste de consciência do próprio desvio de fala e, posteriormente, a avaliação do processamento auditivo.

O teste de consciência do próprio desvio de fala tem por finalidade fazer com que a criança ouça e julgue os desvios existentes em sua própria fala ${ }^{7}$. A aplicação deste teste foi realizada conforme instruções disponibilizadas no trabalho das autoras, como descrito a seguir.

A partir da amostra de fala utilizada na AFC de cada criança, selecionaram-se dez palavras produzidas com desvio, as quais foram editadas e gravadas isoladamente. Os registros foram coletados em ambiente silencioso com o gravador digital Powerpack - Digital Voice Recorder DRV800 III e posteriormente foram armazenados em um computador por meio do programa Recorder V2.0 Digital Voice. Depois de armazenadas, as gravações foram editadas usando o programa GoldWave audio digital editor e a seguir foram apresentadas à criança por meio de fones de ouvidos.

Após aproximadamente uma semana, as palavras foram apresentadas à criança de forma descontextualizada no intuito de dificultar que ela percebesse que se tratava de palavras produzidas por ela mesma. Foi explicado para ela que 
seriam apresentadas palavras faladas "por uma outra criança" e que ela deveria julgar se eram produzidas de forma adequada ou inadequada. Conforme instruções do teste, a pergunta feita para a criança era: Essa criança está falando "direitinho" a palavra?

Foi mostrada uma figura correspondente para cada uma das dez palavras selecionadas, após perguntava-se à criança se a palavra foi ou não produzida "direitinho", e assim, a mesma escutava a gravação para posterior julgamento. As dez palavras produzidas com desvio foram apresentadas duas vezes para que a criança julgasse as palavras produzidas por ela própria. Em momento algum a criança foi informada de que se tratava de palavras produzidas por ela mesma para evitar que fatores emocionais interferissem no teste ${ }^{7}$.

Para os dois julgamentos de cada palavra foram atribuídos valores, sendo 1 (um) ponto para os julgamentos corretos e 0 (zero) para os incorretos. A pontuação máxima possível de ser alcançada foi 20 (vinte).

Para a análise individual dos sujeitos, foi estipulado para esta pesquisa que porcentagens iguais ou maiores que $60 \%$ de acertos nos julgamentos indicariam o estabelecimento da consciência do próprio desvio de fala. Já resultados abaixo de $60 \%$ de acertos foram considerados indicativos de que a criança não teria estabelecida a CPDF. Adotouse a porcentagem de $60 \%$ com base na média do valor máximo (100\%) que poderia ser atingido pelos sujeitos. Este valor denota quantificação da qualidade da consciência do próprio desvio de fala.

Para avaliação do processamento auditivo utilizou-se a Avaliação Simplificada do Processamento Auditivo (Triagem) e os testes especiais de Fala no Ruído (FR) e o Teste de Dissílabos Alternados - SSW, presentes no Processamento Auditivo Central: Manual de Aplicação ${ }^{15}$.

A Triagem consta em três subtestes, onde 0 primeiro, de Sequência de Sons Não Verbais, consiste na utilização de três sequências de quatros instrumentos (côco, agogô, sino e guizo). Para êxito deve-se acertar pelo menos duas sequências. O segundo subteste é o de Sequência de Sons Verbais, onde é falado, pelo examinador, três sequências de palavras monossilábicas (PA, TA, CA) e deve-se responder corretamente pelo menos duas sequências, para êxito. Por fim, o terceiro subteste, Teste de Localização Sonora, onde o examinador, munido do sino, deve tocá-lo em cinco direções ao redor do sujeito a ser examinado (lado direito, lado esquerdo, à frente, atrás e acima da cabeça), e para considerar êxito, deve-se acertar as direções laterais e pelo menos duas das outras direções.
O Teste de Fala no Ruído consiste na aplicação de uma lista de palavras em cada orelha separadamente, e um ruído branco é acrescentado, de forma ipsilateral, em proporção sinal/ruído de 10 dBNS. O Teste Dicótico de Dissílabos Alternados (SSW) envolve uma lista de palavras faladas de forma livre e de forma competitiva - direita livre ou não competitiva (DNC) à direita competitiva (DC) à esquerda competitiva (EC) à esquerda livre ou não competitiva (ENC) (habilidades auditivas de ordenação temporal complexa e figura-fundo para sons verbais).

Este estudo faz parte de um projeto de pesquisa aprovado pelo Comitê de Ética em Pesquisa da Instituição de Ensino Superior de origem (número do cadastro: 23081.006440/2009-60).

Os dados do estudo foram submetidos à análise estatística por meio do programa SAS (Statistical Analisys System) versão 8.02, utilizando-se o Teste Correlação de Pearson. O nível de significância adotado para os testes estatísticos foi de 5\% $(p<0.05)$.

\section{RESULTADOS}

A tabela 1 mostra os resultados obtidos de acordo com os valores de média, desvio padrão (DP) e de mínimo e máximo em todos os testes realizados (consciência do próprio desvio de fala e processamento auditivo).

No que se refere à consciência do próprio desvio de fala, observa-se que o grupo pesquisado obteve uma média de $64 \%$ de acertos, o que demonstra que as crianças com desvio fonológico podem apresentar consciência das trocas realizadas em sua própria fala.

É importante destacar ainda, que todas as crianças apresentaram, no teste de localização sonora, respostas corretas nas tarefas de "localização sonora à direita" e "localização sonora à esquerda", já que uma alteração nestas localizações é sugestiva de alteração neurológica.

A tabela 2 apresenta a correlação entre os resultados dos testes do processamento auditivo e o teste de consciência do próprio desvio de fala. Foram descritos apenas os valores com significância maior ou igual a 5\%.

Nota-se que houve poucas correlações a serem descritas, ao se considerar os valores citados anteriormente. O número reduzido da amostra pode ter influenciado este fator, entretanto, é possível ver que há interferência do processamento auditivo nas possibilidades das crianças com desvios terem consciência destas alterações, como o teste de sequência verbal da Triagem e o SSW. 
Tabela 1 - Valores obtidos nos testes de consciência do próprio desvio de fala e do processamento auditivo

\begin{tabular}{cccccc}
\hline \multicolumn{2}{c}{ Processamento Auditivo } & Média & DP & Mínimo & Máximo \\
\hline \multirow{2}{*}{ CPDF } & & $64 \%$ & & $21 \%$ & $100 \%$ \\
\hline \multirow{2}{*}{ Triagem } & SI & 2,50 & 0,75 & 1,00 & 3,00 \\
& SV & 2,00 & 0,75 & 1,00 & 3,00 \\
& TLS & 3,63 & 1,06 & 2,00 & 5,00 \\
\hline \multirow{2}{*}{ Fala no Ruído } & OD & 87,00 & 7,01 & 80,00 & 96,00 \\
& OE & 88,50 & 8,12 & 80,00 & 100,00 \\
\hline \multirow{2}{*}{ SSW } & DNC & 0,58 & 0,11 & 0,35 & 0,70 \\
& DC & 0,39 & 0,17 & 0,05 & 0,55 \\
& EC & 0,34 & 0,08 & 0,20 & 0,45 \\
& ENC & 0,46 & 0,20 & 0,20 & 0,70 \\
\hline
\end{tabular}

Legenda: DP = Desvio Padrão; CPDF = Consciência do Próprio Desvio de Fala; SI = Sequência instrumental; SV = Sequência verbal; TLS = Teste de Localização Sonora; OD = Orelha Direita; OE = Orelha Esquerda; SSW = Teste Dicótico de Dissílabos Alternados; DNC = Direita Não Competitiva; DC = Direita Competitiva; EC = Esquerda Competitiva; ENC = Esquerda Não Competitiva

Tabela 2 - Correlação entre os testes do processamento auditivo e a consciência do próprio desvio de fala

\begin{tabular}{ccc}
\hline \multicolumn{2}{c}{ Processamento Auditivo } & \multirow{2}{*}{ CPDF } \\
\hline Teste & Subteste & $0,70^{*}$ \\
Triagem & Sequência Verbal & $0,69^{*}$ \\
\hline SSW & Direita Não Competitiva & \\
\hline
\end{tabular}

Legenda: CPDF = Consciência do Próprio Desvio de Fala; SSW = Teste Dicótico de Dissílabos Alternados

${ }^{\star}$ Estatisticamente significante (Correlação de Pearson, com $\mathrm{p}<0,05$ )

\section{DISCUSSÃO}

Observando-se os resultados obtidos, verificase que, mesmo quando o desempenho nos testes do processamento auditivo (Triagem, Fala no Ruído e SSW) não é satisfatório, pode haver consciência do próprio desvio de fala (Tabela 1). Este achado sugere que crianças com desvio fonológico, mesmo apresentando dificuldade para entender ou interpretar o que ouvem, são capazes de perceber os erros que produzem na própria fala.

Tal proposição também foi mencionada por alguns autores, os quais afirmaram que crianças com desvio fonológico parecem ter acesso a representações fonológicas normais, uma vez que se mostraram ser capazes de reconhecer as trocas em sua fala $6,7,9,16,17$.

Por outro lado, de acordo com os valores mínimos e máximos mostrados na tabela 1 , houve valores abaixo de $60 \%$, o que indica que determinados sujeitos, apesar de apresentar consciência do próprio desvio de fala, tal habilidade apresenta- se defasada ou não estabelecida, e não ocorre para todos os fonemas que produz.

Assim como a consciência fonológica, a consciência do próprio desvio de fala é considerada pelos autores deste estudo como uma habilidade metalinguística. Consta na literatura, que o déficit em consciência fonológica pode estar associado a alterações do processamento auditivo, uma vez que a integridade dos mecanismos fisiológicos auditivos tem um papel fundamental na percepção da fala, no aprendizado e na compreensão da linguagem, sendo, consequentemente, pré-requisito na aquisição da leitura e escrita ${ }^{10,18-22}$.

Como se pode observar, ao contrário do que era esperado, crianças com desvio fonológico podem ser capazes de identificar os erros produzidos em sua fala, ainda que não tenham apresentado um bom desempenho em tarefas envolvendo o processamento auditivo. Esses resultados corroboram achados de outra pesquisa em que foi observado que crianças com desvios fonológicos podem obter bons resultados em tarefas de consciência fonoló- 
gica, apesar de apresentar alterações em alguns testes do processamento auditivo ${ }^{23}$.

Ao analisar os resultados da avaliação do processamento auditivo de forma isolada, na Triagem, pode-se constatar, tanto nos subtestes de Sequência Verbal e Sequência Não Verbal, como no subteste de Localização Sonora, alterações do processamento auditivo. Em todos os subtestes foram observados valores mínimos indicativos de tal alteração. É importante destacar que todas as crianças apresentaram respostas corretas nas tarefas que envolvem localizações laterais, o que em situação contrária seria indício de alteração neurológica ${ }^{15,24}$.

No teste de Fala no Ruído, nota-se uma leve superioridade nos valores obtidos à orelha esquerda em relação à orelha direita. Este teste tem por finalidade avaliar a integridade das vias auditivas periféricas, com o resultado obtido pode-se pensar que as crianças deste estudo tendem a ter este hemisfério esquerdo auditivamente mais maduro ${ }^{15,25}$. Seria relevante, no entanto, novas pesquisas para esclarecimento deste achado.

O teste SSW, por sua vez, mostrou resultados bastante inferiores, já que grande parte dos achados, sobretudo nas funções de escuta competitiva, foi abaixo de $50 \%$. O SSW avalia a região cortical, com relação às habilidades de separação binaural e resolução temporal simples. Este achado nos sugere que as crianças avaliadas na presente pesquisa possuem certa dificuldade nestas habilidades, comparadas às crianças com desenvolvimento fonológico típico ${ }^{26,27}$.

Vale ressaltar que a habilidade de separação binaural é processada no corpo caloso, estrutura responsável pela integração dos hemisférios cerebrais e que, em crianças até os 7:0, como as aqui estudadas, pode ainda estar em fase de maturação ${ }^{15,24}$.

Ainda no SSW, vemos que o desempenho geral foi de acordo com a literatura, onde os valores obedeceram a ordem DNC à ENC à DC à EC, onde DNC apresentou os melhores valores, e EC apresentou os piores ${ }^{23,26}$.

Quanto às correlações obtidas neste estudo, somente os testes de Sequência Verbal da triagem, e DNC do SSW, obtiveram valores acima de $50 \%$, com resultado estatisticamente significante (Tabela 2). Em decorrência de ter ocorrido correlação do teste de consciência do próprio desvio de fala somente com o subteste de Sequência Verbal, apesar de serem tarefas distintas de serem realizadas, isso remete ao fato deste ser o único subteste empregado na avaliação do processamento auditivo a envolver vocábulos e sequências linguísticas propriamente ditas, como na consciência do próprio desvio de fala. Já quanto à correlação entre DNC e consciência do próprio desvio de fala, pode-se pensar que esta ocorreu diante de ser uma tarefa mais fácil de ser executada, uma vez que não há competitividade de sons nesta fase do teste.

\section{CONCLUSÃO}

A consciência do próprio desvio de fala pode estar preservada em crianças com desvio fonológico, ainda que alterações em alguns testes do processamento auditivo tenham sido observadas. Os resultados deste estudo demonstram, sobretudo, a necessidade de investigações mais aprofundadas no que se refere à percepção da fala. 


\begin{abstract}
Background: the ability of recognizing the own speech impairment and its possible relationship with the auditory processing in children with phonological disorder. Procedures: this study included eight subjects diagnosed with phonological disorders, age between 5:0;26 and 7:7;2-year old. The phonetic / phonological system was evaluated by the Phonological Assessment of Child Speech. The Awareness of the own Speech Impairment Test, which aims to make the child listening and judging the deviations existing in their own speech, was applied. For evaluating the auditory processing, we used the Simplified Assessment of Auditory Processing (Screening), and Speech in Noise and the Alternate Dichotic Dissyllable Special Tests. Results: all subjects were rated below expectations, with values below $50 \%$ of the maximum possible value in the tests of auditory processing. As for awareness of their own speech impairment, all subjects showed this ability in quite variable values even though their performance in the auditory processing tests was not satisfactory. This finding suggests that children in this period ( 5 to 7 -year old), even the ones with difficulty to understanding or interpreting what they hear, are able to realize the mistakes they have in speech. Conclusion: the awareness of the own speech impairment is found in subjects with phonological disorder, regardless the performance in auditory processing tasks.
\end{abstract}

KEYWORDS: Speech Disorders; Hearing Tests; Auditory Perception; Speech Perception; Child

\section{REFERÊNCIAS}

1. Yavas M, Hernandorena CLM, Lamprecht RR. Avaliação fonológica da criança: reeducação e terapia. Porto Alegre: Artes Médicas, 2001.

2. Wertzner HF, Amaro L, Galea DES. Phonological performance measured by speech severity indices compared with correlated factors. Med J. 2007;125(6):309-14.

3. McLeod S, Isaac K. Use of spectrographic analyses to evaluate the efficacy of phonological intervention. Clin Ling Phon. 1995;9:229-34.

4. Rodrigues LL, Freitas MCC, Albano EC, Berti LC. Acertos gradientes nos chamados erros de pronúncia. Revista de Letras (PPGL / UFSM). 2008;36:85-112.

5. Brasil BC, Melo RM, Mota HB, Dias RF, Mezzomo CM, Giacchini V. O uso da estratégia de alongamento compensatório em diferentes gravidades do desvio fonológico. Rev Soc Bras Fonoaudiol. 2010;15(2):231-7.

6. Magnusson E. Consciência metalingüística em crianças com desvios fonológicos. In: Yavas MS. (Org.). Desvios fonológicos em crianças: Teoria, pesquisa e tratamento. Mercado Aberto. Porto Alegre, 1990. p.109-48.

7. Menezes G, Lamprecht RR. A consciência fonológica na relação fala-escrita em crianças com Desvio Fonológico Evolutivo (DFE). Letras de Hoje. 2001;36(3):743-9.

8. McGregor KK, Schwartz RG. Converging evidence for underlying phonological representation in a child who misarticulates. J. Speech Hear Res. 1992;35:596-603.

9. Dias RF, Mota HB, Mezzomo CL. Desvio Fonológico: aspectos sobre produção, percepção e escrita.RevSocBrasFonoaudiol.2010;15(4):554-60.

10. Neves IF, Schochat E. Maturação do processamento auditivo em crianças com e sem dificuldades escolares. Pró-Fono $\mathrm{R}$ Atual Cient. 2005;17(3):311-20.

11. Chermak GD, Silva ME, Nye J, Hasbrouck J, Musiek FE. An update on professional education and clinical practices in central auditory. J Am Acad Audiol. 2007;18(5):428-52.

12. Muniz LF, Roazzi A, Schochat E, Teixeira CF, Lucena JA. Avaliação da habilidade de resolução temporal, com uso do tom puro, em crianças com e sem desvio fonológico. Rev CEFAC. 2007;9(4):550-62.

13. Engelmann L, Ferreira MIDC. Avaliação do processamento auditivo em crianças com dificuldades de aprendizagem. Rev Soc Bras Fonoaudiol. 2009;14(1):69-74.

14. American Speech-Language-Hearing Association. (2005). (Central) Auditory Processing Disorders [Technical Report].

15. Pereira LD, Schochat E. Processamento Auditivo Central: Manual de Aplicação. São Paulo: Lovise, 1997.

16. Dias RF, Mota HB, Mezzomo CL. A consciência fonológica e a consciência do próprio desvio de fala nas diferentes gravidades do desvio fonológico. Rev CEFAC. 2009;11(4):561-70. 
17. Rizzon GF, Chiechelski P, Gomes E. Relação entre consciência fonológica e desvio fonológico em crianças da $1^{\underline{a}}$ série do ensino fundamental. Rev CEFAC. 2009; 11(supl2):201-7.

18. Watson BU, Miller T. Auditory perception, phonological processing and reading ability/ disabilities. J Speech Hear Res 1993;36:850-63.

19. Bishop DV, Carlyon RP, Deeks JM, Bishop SJ. Auditory temporal processing impairment: neither necessary nor sufficient for causing language impairment in children. J Speech Lang Hear Res. 1999;42(6):1295-310.

20. Heath SM, Hogben JH, Clark CD. Auditory temporal processing in disabled readers with and without oral language delay. J Child Psychol Psychiatry. 1999;40(4):637-47.

21. Garcia VL, Campos DBKP; Padovani CR. Associação entre a avaliação de habilidades de consciência fonológica e de processamento auditivo em crianças com e sem distúrbio de aprendizagem. Fono Atual. 2005;8(31):4-11.

22. Boets $B$, Wouters $J$, van Wieringen $A$, Ghesquière
P. Auditory processing, speech perception and phonological ability in pre-school children at highrisk for dyslexia: a longitudinal study of the auditory temporal processing theory. Neuropsychologia. 2007;45(8):1608-20.

23. Quintas VG, Attoni TM, Keske-Soares M, Mezzomo CL. Processamento auditivo e consciência fonológica em crianças com aquisição de fala normal e desviante. Pró-fono R Atual Cient. 2010;22(4):497-502.

24. Pereira LD. Sistema Auditivo e desenvolvimento das habilidades auditivas. In: Ferreira, LP, BefiLopes, DM, Limonge, SCO. Tratado de Fonoaudiologia. Roca: São Paulo; 2004.

25. Springer SP, Deutsch G. Cérebro Esquerdo, Cérebro Direito. São Paulo: Summus, 1993. 412 p.

26. Araujo NSS, Ruiz ACP, Pereira LD. SSW-analise qualitativa dos erros: inventário de atendimento de 2005. Rev CEFAC. 2009;11(supl1):44-51.

27. Caumo DTM, Ferreira MIDC. Relação entre desvios fonológicos e processamento auditivo. Rev Soc Bras Fonoaudiol. 2009;14(2):234-40.

http://dx.doi.org/10.1590/S1516-18462011005000037

RECEBIDO EM: 28/09/2010

ACEITO EM: 07/02/2011

Endereço para correspondência:

Roberta Michelon Melo

Rua Tuiuti, no 1850 , apto 501A, Centro

Santa Maria - RS - Brasil

CEP: 97015-662

E-mail: roberta_m_melo@ hotmail.com 